\title{
Nucleosynthesis in jet-induced supernovae
}

\author{
Nozomu Tominaga*t \\ Department of Physics, Faculty of Science and Engineering, Konan University \\ 8-9-1 Okamoto, Kobe, Hyogo 658-8501, Japan \\ E-mail: tominaga@konan-u.ac.jp
}

The first metal enrichment in the universe was made by supernova (SN) explosions of population III stars and the results are recorded in abundance patterns of extremely metal-poor (EMP) stars. Meanwhile, it has been found that gamma-ray bursts with relativistic jets are associated with highly-energetic SNe (hypernovae) and observations of SNe in recent days also smoke out that SNe are universally aspherical. Thus, I present hydrodynamical and nucleosynthetic properties of the jet-induced explosion of a population III star with a two-dimensional special relativistic hydrodynamical code. In the jet-induced $\mathrm{SNe}$, Fe-peak products are ejected along the jet axis, while unprocessed materials fall onto a central remnant along the equatorial plane. This coexistence accounts for the abundance patterns of the EMP stars. Also, the jet-induced explosion realizes the high-entropy environment that enhances $[(\mathrm{Sc}, \mathrm{Ti}, \mathrm{V}, \mathrm{Cr}, \mathrm{Co}, \mathrm{Zn}) / \mathrm{Fe}]$. The enhancements of $[\mathrm{Sc} / \mathrm{Fe}]$ and $[\mathrm{Ti} / \mathrm{Fe}]$ improve agreements with the abundance patterns of the EMP stars. Furthermore, I point out that the evidence of jet-induced SN is found in a Si-deficient metal-poor star HE 1424-0241 with high $[\mathrm{Mg} / \mathrm{Si}](=1.4)$ and normal $[\mathrm{Mg} / \mathrm{Fe}](=0.4)$. While the peculiar abundance pattern is difficult to be reproduced by previous SN models, it can be reproduced only by the angle-delimited yield of the jet-induced SN if the interaction between the SN ejecta and interstellar medium induces a weak mixing of the abundances.

11th Symposium on Nuclei in the Cosmos, NIC XI

July 19-23, 2010

Heidelberg, Germany

*Speaker.

${ }^{\dagger}$ The appointed researcher of the Institute for the Physics and Mathematics of the Universe. 


\section{Introduction}

In the early universe, the enrichment by a single supernova (SN) can dominate the preexisting metal contents (e.g., [1]). The Pop III SN shock compresses the SN ejecta consisting of heavy elements, e.g., $\mathrm{O}, \mathrm{Mg}, \mathrm{Si}$, and $\mathrm{Fe}$, and the circumstellar materials consisting of $\mathrm{H}$ and $\mathrm{He}$. The abundance pattern of the enriched gas reflects nucleosynthesis in the $\mathrm{SN}$ and the second-generation stars will be formed from the enriched gases. Thus the abundances of the second generation stars preserve the information of nucleosynthesis in Pop III SNe [2].

Present-day photometric and spectroscopic observations indicate that gamma-ray bursts (GRBs) and $\mathrm{SNe}$ are aspherical explosions with jet(s) (e.g., [3, 4, 5]). Such aspherical SN explosions are also suggested in the early universe by the abundance patterns of extremely metal-poor (EMP) stars with $[\mathrm{Fe} / \mathrm{H}]<-3 .{ }^{1}$ The $\mathrm{C}$-enhanced type of the EMP stars have been well explained by the faint $\mathrm{SNe}[6,7]$, except for their large $\mathrm{Co} / \mathrm{Fe}$ and $\mathrm{Zn} / \mathrm{Fe}$ ratios (e.g., [8]). The enhancement of $\mathrm{Co}$ and $\mathrm{Zn}$ in low metallicity stars requires explosive nucleosynthesis under high entropy. In a spherical model, a high entropy explosion corresponds to a high energy explosion that inevitably synthesizes a large amount of ${ }^{56} \mathrm{Ni}$. Thus, it was suggested that some faint $\mathrm{SNe}$ are associated with a narrow jet within which a high entropy region is confined [9].

In this paper, I present hydrodynamical and nucleosynthetic models of the jet-induced explosions of a $40 M_{\odot}$ star and show that only an angular-delimited yield of a jet-induced SN explosion can account for an abundance pattern of a peculiar Si-deficient EMP star. Furthermore, I compare the jet-induced explosion with the spherical SN model applied the mixing-fallback model [10] and connect properties of the jet-induced explosion to the mixing-fallback model.

\section{Models}

I investigate a jet-induced SN explosion of a Pop III $40 M_{\odot}$ star with a two-dimensional relativistic Eulerian hydrodynamic and nucleosynthesis calculation (see [11, 12] for the model detail). Because the explosion mechanism of GRB-associated SNe is still under debate, I do not consider how the jet is launched but deal the jet parametrically with the following five parameters: energy deposition rate $\left(\dot{E}_{\mathrm{dep}}\right)$, total deposited energy $\left(E_{\mathrm{dep}}\right)$, initial half angle of the jets $\left(\theta_{\mathrm{jet}}\right)$, initial Lorentz factor $\left(\Gamma_{\text {jet }}\right)$, and the ratio of thermal to total deposited energies $\left(f_{\text {th }}\right)$. The jet is injected from the inner boundary at an enclosed mass $M_{0}$ corresponding to a radius $R_{0}$. In this paper, I show two models; (A) a model with $\dot{E}_{\mathrm{dep}, 51}=\dot{E}_{\mathrm{dep}} /\left(10^{51} \mathrm{ergs} \mathrm{s}^{-1}\right)=120$ and $M_{0}=1.4 M_{\odot}\left(R_{0}=900\right.$ $\mathrm{km})$ and (B) a model with $\dot{E}_{\mathrm{dep}, 51}=120$ and $M_{0}=2.3 M_{\odot}\left(R_{0}=2700 \mathrm{~km}\right)$. The other parameters are same for each model; $E_{\mathrm{dep}}=1.5 \times 10^{52} \mathrm{ergs}, \theta_{\text {jet }}=15^{\circ}, \Gamma_{\text {jet }}=100$ and $f_{\mathrm{th}}=10^{-3}$. The mass of jets is $M_{\text {jet }} \sim 8 \times 10^{-5} M_{\odot}$.

\section{Results}

The hydrodynamical calculations are followed until the homologously expanding structure is reached $(v \propto r)$. Then, the ejected mass elements are identified from whether their radial velocities

\footnotetext{
${ }^{1}$ Here $[\mathrm{A} / \mathrm{B}]=\log _{10}\left(N_{\mathrm{A}} / N_{\mathrm{B}}\right)-\log _{10}\left(N_{\mathrm{A}} / N_{\mathrm{B}}\right) \odot$, where the subscript $\odot$ refers to the solar value and $N_{\mathrm{A}}$ and $N_{\mathrm{B}}$ are the abundances of elements $\mathrm{A}$ and $\mathrm{B}$, respectively.
} 

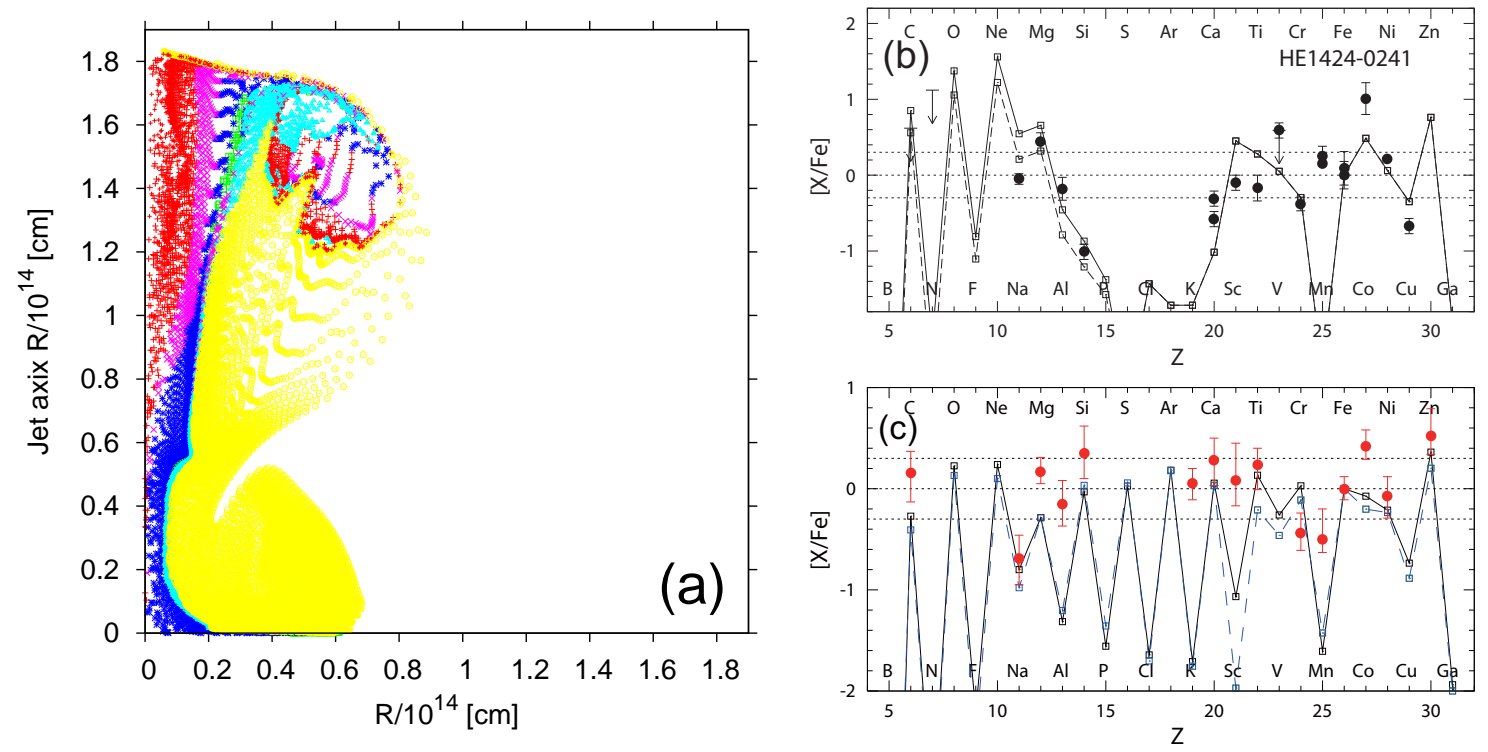

Figure 1: (a) Positions of the mass elements at $t=10^{5} \mathrm{~s}$ for model A. Symbols of the marks represents the abundance of the mass element ( $\mathrm{H}$ : circle, He: triangles, $\mathrm{O}+\mathrm{C}$ : squares, $\mathrm{O}+\mathrm{Mg}$ : stars, $\mathrm{Si}$ : crosses, and $\mathrm{Fe}$ : pluses). Size of the marks represents the origin of the mass element (the jet: dots, and the shocked stellar mantle: filled circles). (b) Comparison between the abundance pattern of HE 1424-0241 (filled circles) and the angle-delimited yields of model $\mathrm{A}$ for $30^{\circ} \leq \theta<40^{\circ}$ (solid line) and $30^{\circ} \leq \theta<35^{\circ}$ (dashed line). (c) Comparison of the abundance patterns of model B (solid line), the EMP stars (filled circles, [13]), and the mixing-fallback models with $M_{\text {cut }}($ ini $)=2.3 M_{\odot}, M_{\text {mix }}($ out $)=10.3 M_{\odot}$ and $f=0.27$ (dashed line).

exceed the escape velocities at their positions. Figures 1a shows the abundance distribution at $t=10^{5} \mathrm{~s}$ for model A. I classify the mass elements by their abundances as follows: (1) Fe with $X\left({ }^{56} \mathrm{Ni}\right)>0.04$, (2) Si with $X\left({ }^{28} \mathrm{Si}\right)>0.08$, (3) $\mathrm{O}+\mathrm{Mg}$ with $X\left({ }^{16} \mathrm{O}\right)>0.6$ and $X\left({ }^{24} \mathrm{Mg}\right)>0.01$, (4) $\mathrm{O}+\mathrm{C}$ with $X\left({ }^{16} \mathrm{O}\right)>0.6$ and $X\left({ }^{12} \mathrm{C}\right)>0.1$, (5) He with $X\left({ }^{4} \mathrm{He}\right)>0.7$, and (6) $\mathrm{H}$ with $X\left({ }^{1} \mathrm{H}\right)>0.3$. If a mass element satisfies two or more conditions, the mass element is classified into the class with the smallest number.

\subsection{Abundance patterns of the metal-poor stars}

A very peculiar, Si-deficient, metal-poor star HE 1424-0241 was observed [14]. Its abundance pattern with high $[\mathrm{Mg} / \mathrm{Si}](\sim 1.4)$ and normal $[\mathrm{Mg} / \mathrm{Fe}](\sim 0.4)$ is difficult to be reproduced by previous $\mathrm{SN}$ models. This is because $\log \left\{[X(\mathrm{Mg}) / X(\mathrm{Si})] /[X(\mathrm{Mg}) / X(\mathrm{Si})]_{\odot}\right\} \lesssim 1.6$ is realized in the $\mathrm{O}+\mathrm{Mg}$ layer at the presupernova stage (e.g., $[15,9]$ ). Thus, in order to reproduce the abundance pattern of HE 1424-0241, the SN yield is required to include explosively-synthesized Fe but not explosively-synthesized Si.

The angle-delimited yield may possibly explain the high $[\mathrm{Mg} / \mathrm{Si}]$ and normal $[\mathrm{Mg} / \mathrm{Fe}]$ (Fig. 1b). Figure $1 \mathrm{~b}$ shows that the yields integrated over $30^{\circ} \leq \theta<40^{\circ}$ and $30^{\circ} \leq \theta<35^{\circ}$ of model A reproduce the abundance pattern of HE 1424-0241. The yield consist of Mg in the inner region and $\mathrm{Fe}$ in the outer region (Fig. 1a). Although there are some elements to be improved, the elusive feature of HE 1424-0241 could be explained by taking into account the angular dependence of the yield. The high $[\mathrm{Mg} / \mathrm{Si}]$ and normal $[\mathrm{Mg} / \mathrm{Fe}]$ can be realized with an appropriate integration range 
if the Fe mass elements penetrate the stellar mantle (i.e., the duration of the jet injection is long) and if the $\mathrm{O}+\mathrm{Mg}$ mass elements are ejected in all directions (i.e., $\dot{E}_{\text {dep }}$ is high).

\subsection{Comparison with the spherical supernova model}

The calculations of the jet-induced explosions show that the ejection of the inner matter is compatible with the fallback of the outer matter. This is consistent with the two-dimensional illustration of the mixing-fallback model (Fig. 12b in [16]).

I clarify the relation between the mixing-fallback model and the jet-induced explosion model by comparing the yields. The mixing-fallback model has three parameters; initial mass cut $\left[M_{\text {cut }}(\right.$ ini) $]$, outer boundary of the mixing region $\left[M_{\operatorname{mix}}\right.$ (out)], and a fraction of matter ejected from the mixing region $(f)$. The remnant mass is written as $M_{\text {rem }}=M_{\text {cut }}($ ini $)+(1-f)\left[M_{\text {mix }}\right.$ (out) $-M_{\text {cut }}$ (ini) $]$. The three parameters would relate to the hydrodynamical properties of the jet-induced explosion models, e.g., the inner boundary $\left(M_{0}\right)$, the outer edge of the accreted region $\left(M_{\mathrm{acc}, \text { out }}\right)$, and the width between the edge of the accreted region and the jet axis.

The angle-integrated yield of model B is compared with the yields of the spherical SN model with $M_{\mathrm{ms}}=40 M_{\odot}$ and $E_{\mathrm{dep}}=3 \times 10^{52}$ ergs. The inner boundary, the outer edge of the accreted region, and the central remnant mass of model B are $M_{0}=2.3 M_{\odot}, M_{\mathrm{acc}, \text { out }}=12.2 M_{\odot}$, and $M_{\mathrm{rem}}=$ $8.1 M_{\odot}$. For the spherical SN model, the explosion energy is deposited instantaneously as a thermal bomb and I set $M_{\text {cut }}\left(\right.$ ini) to be the same as $M_{0}$ [i.e., $M_{\text {cut }}($ ini $)=2.3 M_{\odot}$ ] and apply $M_{\text {mix }}($ out $)=$ $10.3 M_{\odot}$ and $f=0.27$. $f$ is the fraction of the solid angle of the Si-burning region (hereafter the fraction is written as $\left.f_{\mathrm{Si}}\right)$. $M_{\text {mix }}$ (out) is set to yield the same $M_{\text {rem }}$ as model B. The angle-integrated abundance pattern of model B is roughly reproduced by the mixing-fallback model (Fig. 1c). Thus, I conclude that the jet-induced explosion is approximated by the mixing-fallback model reasonably. $M_{0}, f_{\mathrm{Si}}$, and $M_{\text {rem }}$ in the jet-induced explosion model are represented by $M_{\text {cut }}\left(\right.$ ini), $f$, and $M_{\text {rem }}$ in the mixing-fallback model, respectively.

There are some elements showing differences, Sc, Ti, V, Cr, Co, and $\mathrm{Zn}$. The enhancements of $[\mathrm{Sc} / \mathrm{Fe}]$ and $[\mathrm{Ti} / \mathrm{Fe}]$ improve agreements with the observations. The differences stem from the highentropy explosion due to the concentration of the energy injection in the jet-induced explosion (e.g., [17]). Such thermodynamical features of the jet-induced explosion model cannot be reproduced by the mixing-fallback model exactly but a "low-density" modification might mimic the high-entropy environment (e.g., $[9,16])$.

\section{Conclusions and Discussion}

I present the aspherical abundance distributions and investigate the angular dependence of the yield. The abundance distributions in the jet-induced SN ejecta could be examined by spatiallyresolved observations of supernova remnants (e.g., [18]). The angle-delimited yield could reproduce the extremely peculiar abundance pattern of HE 1424-0241. However, the angle-delimited yields of model A have a large scatter that may be inconsistent with the relatively small scatter in the abundance ratios of the EMP stars. This implies that the angular dependence of the yield in most $\mathrm{SNe}$ is diluted by the strong mixing of the SN ejecta. The angle-delimited yield strongly depends on which mass elements are included into the integration. This would be determined by the abundance mixing in the SN ejecta [19] and by the region where the next-generation star takes in 
the metal-enriched gas. To investigate this issue further, it is required to calculate three-dimensional evolution of the supernova remnant in the ISM.

The angle-integrated yield of the jet-induced explosion is well reproduced by a spherical SN model applied the mixing-fallback model. This confirms that the mixing-fallback model approximates the jet-induced explosion well and that the mixing and fallback in hypernovae assumed in the mixing-fallback model are actually achieved in aspherical explosions. The abundance ratios between elements synthesized in different regions (e.g., $\mathrm{C}, \mathrm{O}, \mathrm{Mg}$, and $\mathrm{Fe}$ ) depend on the hydrodynamical structure of the explosion, e.g., the fallback. Thus, such macroscopic properties of the jetinduced explosion are represented by the mixing-fallback model. In particular, $M_{0}, f_{\mathrm{Si}}$, and $M_{\mathrm{rem}}$ in the jet-induced explosion model are represented by $M_{\text {cut }}$ (ini), $f$, and $M_{\text {rem }}$ in the mixing-fallback model, respectively. On the other hand, the ratios between the explosively-synthesized elements depend on the thermodynamical properties of the explosion. In particular, [Sc/Fe], [Ti/Fe], [V/Fe], $[\mathrm{Cr} / \mathrm{Fe}],[\mathrm{Co} / \mathrm{Fe}]$ and $[\mathrm{Zn} / \mathrm{Fe}]$ are enhanced by the high-entropy environment in the jet-induced explosion, thus showing differences from the mixing-fallback model. The enhancement of [Sc/Fe] and $[\mathrm{Ti} / \mathrm{Fe}]$ improve the agreement with the observations.

\section{References}

[1] J. Audouze \& J. Silk, J., ApJ 451 (1995) L49

[2] T. C. Beers \& N. Christlieb, ARA\&A 43 (2005) 531

[3] D. A. Frail, et al., ApJ 562 (2001) L55

[4] K. Maeda, et al., ApJ 565 (2002) 405

[5] K. Maeda, et al., Science 319 (2008) 1220

[6] H. Umeda \& K. Nomoto, Nature 422 (2003) 871

[7] N. Iwamoto, H. Umeda, N. Tominaga, K. Nomoto, K. Maeda, Science 309 (2005) 451

[8] E. Depagne, et al., $A \& A 390$ (2002) 187

[9] H. Umeda \& K. Nomoto, ApJ 619 (2005) 427

[10] H. Umeda \& K. Nomoto, ApJ 565 (2002) 385

[11] N. Tominaga, et al., ApJ 657 (2007) L77

[12] N. Tominaga, ApJ 690 (2009) 526

[13] R. Cayrel, et al., $A \& A 416$ (2004) 1117

[14] J. G. Cohen, et al., ApJ 659 (2007) L161

[15] S. E. Woosley \& T. A. Weaver, ApJS 101 (1995) 181

[16] N. Tominaga, H. Umeda, \& K. Nomoto, ApJ 660 (2007) 516

[17] K. Maeda \& K. Nomoto, ApJ 598 (2003) 1163

[18] U. Hwang, et al., ApJ 615 (2004) L117

[19] N. Nakasato \& T. Shigeyama, ApJ 541 (2000) L59 